\title{
Meta
}

Journal des traducteurs

Translators' Journal

\section{BELL, Roger T. (1991) : Translation and Translating. Theory and Practice, Londres/New York, Longman, coll. Applied Linguistic and Language Study, 298 p.}

\section{Yves Gambier}

Volume 38, numéro 3, septembre 1993

URI : https://id.erudit.org/iderudit/002801ar

DOI : https://doi.org/10.7202/002801ar

Aller au sommaire du numéro

Éditeur(s)

Les Presses de l'Université de Montréal

ISSN

0026-0452 (imprimé)

1492-1421 (numérique)

Découvrir la revue

Citer ce compte rendu

Gambier, Y. (1993). Compte rendu de [BELL, Roger T. (1991) : Translation and Translating. Theory and Practice, Londres/New York, Longman, coll. Applied Linguistic and Language Study, 298 p.] Meta, 38(3), 537-542.

https://doi.org/10.7202/002801ar d'utilisation que vous pouvez consulter en ligne.

https://apropos.erudit.org/fr/usagers/politique-dutilisation/ 
-1 BELL, Roger T. (1991): Translation and Translating. Theory and Practice, Londres/ New York, Longman, coll. Applied Linguistics and Language Study, 298 p.

Les rapports entre la linguistique (théorique et appliquée) et la traduction n'ont jamais été faciles et cela dès les années 60 ( $c f$. Catford, Mounin...). Depuis moins de dix 
ans, les références s'accroissent sur les apports possibles de la première à la seconde, plus rarement hélas! l'inverse, en même temps que tente de s'affirmer une traductologie comme champ autonome mais aussi interdisciplinaire. On ne peut donc lire l'ouvrage de Bell, en oubliant par exemple les travaux de Larson 1984: Meaning-base Translation, de Neubert 1985: Text and Translation, d'Arcaini 1986: Analisi Linguistica e Traduzione, de Svejcer 1987: Übersetzung und Linguistik (original russe: 1973), de Malone 1988: The Science of Linguistics in the Art of the Translation, d'Hatim et Mason 1990: Discourse and the Translator, de Nord 1991: Text Analysis in Translation (original allemand: 1988), de Gutt 1991 : Translation and Relevance. Cognition and Context ...pour ne citer que des livres, sans signaler l'abondance d'articles, d'exposés de conférences traitant de stylistique, de sémantique, d'analyse de discours, etc. dans la perspective de la traduction comme processus ou comme matière à enseigner.

Les rejets péremptoires de la linguistique ne devraient donc plus avoir cours — sauf pour quelques-uns qui s'entêtent à croire que la linguistique s'est arrêtée au structuralisme, est bornée à l'analyse de la «langue» comme système formel. Malgré tout, l'intégration des récents courants de la linguistique à la réflexion en traduction reste problématique. Certes des notions comme acceptabilité, cohérence, progression thématique, fonction textuelle, actes de langage... semblent avoir passé dans le discours des deux disciplines. Hatim et Mason (1990) est un exemple récent de synthèse des approches en analyse de discours, en linguistique textuelle, en pragmatique... considérées comme pertinentes pour expliquer le travail du traducteur.

Bell se veut aussi un essai pour rapprocher linguistique et traduction, les malentendus entre les deux étant à l'origine, selon l'auteur, des échecs pour élaborer une théorie de la traduction (p. XVI). Certaines prises de position pour forcer le rapprochement susciteront sans doute l'irritation: pourquoi d'emblée, sans justification, catégoriser la traduction comme part de la linguistique appliquée (p. XI, p. 1, p. 28), affirmer que la traductologie en est encore au stade préscientifique de l'anecdote (p. 3), être «convaincu» (p. 13, p. 32) des nécessités de se tourner vers la psychologie et la linguistique sous prétexte que l'activité traductionnelle est mentale et langagière?

Le livre est à la fois modeste, par son refus de prêcher ou de prescrire, et ambitieux, dans son désir de couvrir la traduction comme processus en dégageant les acquis en pragmatique, en psycholinguistique, en intelligence artificielle... Il ne s'agit plus de s'interroger sur les rapports entre langue et vision du monde, entre structures des langues et psychologie, entre spécificités langagières et universaux : les sciences dites cognitives, les recherches en traduction automatique, les analyses de l'interculturel ont rendu obsolètes bien des débats d'il y a trente ans.

Qu'est-ce que le livre apporte donc de nouveau? Ne reprend-il pas certaines grandes lignes de la linguistique textuelle, de la psycholinguistique... sur lesquelles il y a déjà consensus ? En quoi permet-il à la théorisation de la traduction d'avancer? Ces questions trahissent un doute.

Le livre est, comme les autres titres de la collection, à laquelle il appartient, clairement structuré: il multiplie les introductions (générale, des trois parties, des 7 chapitres), les résumés et synthèses (à la fin de chaque chapitre et de ses sous-ensembles), les renvois - ce qui ne peut exclure certaines répétitions, souvent délibérées, parfois lassantes: par exemple en 2.2, sur le processus non linéaire mais en cascade, au chapitre 4 , sur les macrofonctions, dans la partie 3. Certaines figures, nulle part listées, récapitulent de longs développements : ainsi 2.2 (p. 59), 2.6 (p. 69), 4.2 (p. 122), 4.8 (p. 155), 5.1 (p. 185), 6.3 (p. 214) et 7.2 (p. 236). Il est dommage que dans une présentation si pédagogique l'index des mots clés accumule à l'envi les détours internes plus ou moins adéquats - par exemple «semantic representation: see translation sense, see meaning as semantic sense»: le 
premier est absent, le second ne recouvre pas vraiment la «représentation sémantique»; une lacune étonnante : «sociolinguistique» est enregistré, avec plusieurs sous-entrées, mais pas du tout «psycholinguistique». Quelques absences malheureuses aussi, dans la bibliographie, d'auteurs cités pourtant en notes: Kjolseth 1972, Johnson et Whitelock 1987, Carbonell et Masaru 1987, Riley 1985.

Se présentant comme linguiste descriptiviste (p. 12) et non comme traducteur ou traductologue, l'auteur a su rendre son texte aisé à lire, malgré l'éclectisme des notions, des problèmes abordés. Maîtrisant sa matière, il a évité l'inflation des définitions, des taxinomies qui rend par exemple le style d'Hatim et Mason, traducteurs, plutôt compact sinon confus.

Pour esquisser les types de connaissances et d'aptitudes, jugés indispensables lors de la traduction, et parvenir à modéliser l'activité traductionnelle, Bell propose trois parties reconnues par lui-même comme «inégales» (p. XVII): d'abord (I) justifiant son modèle de la traduction-processus dans sa nécessité (chapitre 1) puis le présentant en bref (chapitre 2), il tente ensuite de le légitimer linguistiquement, l'accent étant mis sur la signification (II), et psycholinguistiquement, dans les manières de générer les textes (III).

Parvient-il à intégrer les modèles linguistiques du sens et de la siginification et les modèles psycholinguistiques de la mémoire et du traitement de l'information? On peut hésiter à l'affirmer. N'a-t-on pas plutôt juxtaposition, les éléments suggérés intervenant d'ailleurs à des niveaux différents de la production et de l'interprétation textuelles? Surtout ces modèles n'éclairent pas le processus visé, supposé être constitué de deux volets (p. 20): l'analyse syntaxique, sémantique et pragmatique du texte LD en un «message» (p. 19), en une «idée» (p. 57), enregistrée comme représentation abstraite, puis la synthèse c'est-à-dire "prendre l'idée» (p. 57) à travers les filtres pragmatique, sémantique et syntaxique pour aboutir au texte LA. Les deux volets sont donc conjoints par une étape intermédiaire de déverbalisation, les trois étant selon Bell des opérations mentales, mémorielles. On ne peut guère s'étonner que peu de choses soient avancées sur la «représentation sémantique» (p. 44, p. 56, exemple p. 63) - même si d'autres auteurs ont proposé une hypothèse similaire dans leur tripartition (compréhension - déverbalisation ou essence de l'énoncé - réexpression): la «boîte noire» du cerveau reste encore largement inconnue et les rapports entre langue et pensée, idéation n'ont cessé d'alimenter des polémiques depuis deux siècles. Par contre, on ne cesse d'être surpris quand, dans un livre qui cherche à circonscrire le processus de traduction, quasiment rien n'est exposé sur les conditions et les contraintes du transfert interlinguistique dont sept modes sont rappelés (p. 70) - comme si le traducteur était deux fois monolingues: en lisant son original et en rédigeant sa traduction (produit) - cf. chapitre 6.3. C'est finalement ce sentiment qui l'emporte au fur et à mesure que la lecture avance: la traduction est définie de manière très restrictive, son processus a été réduit au traitement des textes. La surprise ne peut qu'être renforcée par les exemples choisis, devant illustrer assertions et propositions: ils sont très peu nombreux (même si une quinzaine de langues sont citées), fabriqués pour la plupart, ne dépassant pas les limites de la phrase - sauf l'exemple littéraire cherchant à démontrer le bien-fondé du modèle de la traduction (2.3.1, pp. 60-67). Comment Bell peut-il alors affirmer à la fois que la théorie de la traduction en est restée ou presque aux mêmes expédients et aux mêmes prescriptions énoncés par Tytler en 1791 (pp. 10-11) en partie parce qu'elle a été dominée par des littéraires et que cet état perdure alors qu'une «immense proportion» de traductions aujourd'hui sont techniques, médicales, juridiques, administratives (p. 5)? On ne peut pas dire que le choix de Kipling, de Valéry, de Pouchkine... coïncide avec les déclarations de l'auteur... La différence est ici patente avec Hatim et Mason, soucieux de travailler avec des textes, avec des documents qui ont dû être traduits. 
Sur les options ou décisions du traducteur qui doit «préserver» ou «altérer» (p. 58, p. 68) la visée de l'original, sa structure thématique, son style, presque rien n'est suggéré : tout ne se passe-t-il que dans la tête de ce traducteur? Le donneur d'ouvrage n'a-t-il jamais son mot à dire? Les récepteurs ciblés n'orientent-ils jamais les choix à faire, les solutions à trouver?

Outre la «représentation sémantique», d'autres concepts manquent de précision ou sont trop vite pris comme allant de soi. Par exemple, l'unité de traduction assimilée à la proposition, sous prétexte de «bonne» (p. 29) ou de «considérable évidence» (p. 223). Par exemple, la confusion entre théorie et modèle : celui-ci est-il vulgarisation (p. 23), procédure de présentation (p. 24) ou formulation (p. 25, p. 235) de celle-là ? L'un est-il description, 1'autre explication (p. 26) ? Pourquoi finalement plaider pour une «approche», plus réalisable qu'une «théorie» (p. 27)?

Un regard systématique sur les chapitres confirme les travers et les faiblesses signalés.

Dans I (Model), chapitre 1 (Perspectives on Translation) - 1.1.1 (pp. 9 et 22), le seul avocat du diable appelé à la barre est Newmark, le prédécesseur de Bell à la Polytechnics de Londres: est-ce suffisant pour dénier toute validité aux projets actuels de théorisation? En tout cas, l'absence de toute référence par exemple aux recherches de langue allemande, à d'autres travaux sur la traduction comme processus psycholinguistique (Lörscher, Hönig, Gerloff, Krings, Séguinot, Tirkkonen-Condit...) autorise à contredire l'auteur quand il écrit (p. 10) qu'à part Catford et Nida, dans les années 60 , la théorie n'a guère eu de défenseurs fertiles (il se souvient p. 22 de S. Basnett). D'où parle-t-il (p. 21) pour rejeter autant les fausses interprétations des linguistes à propos de la traduction que les ignorances linguistiques des théoriciens de la traduction?

La partie II (Meaning) est la plus développée pour bien marquer «la centralité du sens dans la traduction» (p. XVII). Mais en quoi est-ce utile d'opérer un détour par la sémantique linguistique traditionnelle - de niveau «langue», dénoncée (p. XVIII) comme une «vision plutôt conservatrice»? Quels rapports à la traduction ou à sa théorisation peut-on établir avec l'analyse componentielle, le champ lexical, les postulats de sens, les prototypes (chapitre 3)? Ces rapports sont à peine esquissés (p. 90) ou restent superficiels: ainsi, la théorie de la référence aiderait à expliquer le rappel d'un mot dans telle langue (p. 86), les oppositions pour traiter des synonymes, des antonymes ne poseraient «aucun problème majeur» au traducteur (p. 94), les ensembles lexicaux n'ont que des frontières floues (p. 102). Avec le chapitre 4 (Logic, grammar, rhetoric), on passe de la forme aux macrofonctions du langage (idéationnelle, interpersonnelle et textuelle) : ici encore, quels rapports faut-il envisager avec la problématique de la traduction? On a l'impression que de la traduction on a glissé vers une approche contrastive des langues (cf. pp. 134, 146, 157, 176...).

Ces deux chapitres ainsi que le cinq (Text and Discourse) éclairent les compétences nécessaires du «bon» traducteur (définies en 2.1) mais guère son activité même! Le chapitre 5 assimile d'ailleurs de facto le traducteur à un lecteur monolingue: les données de linguistique textuelle et de pragmatique qui sont présentées de manière systématique et cohérente s'appliquent à toute forme de communication et non à la traduction en particulier. Cela suffit-il de déclarer que «le parallèle avec la traduction est clair» (p. 182) ? Le statut de linguiste revendiqué par Bell justifie-t-il que la théorie de la traduction soit annexée, sans autre procès, à la linguistique (p. 172), que l'analyse syntaxique soit beaucoup plus ample que celles sémantique et pragmatique (en 2.2), que la sémantique traditionnelle (chapitre 3) ait autant de place que la signification ou sens en situation - occasion d'aborder les standards de la textualité, les actes de langage, le principe de coopération, les paramètres stylistiques (chapitre 5)? 
Avec la partie III (Memory), on retrouve ces mêmes défauts ou manquements. Ainsi au chapitre 6 sur le traitement des textes, on retombe dans du déjà connu avec les typologies (6.1), problème pourtant «particulièrement significatif» (en quoi ?) pour le traducteur (p. 226) ?; avec les éléments de la «compétence linguistique» (6.2), reformulation délibérée de la triple distinction donnée au chapitre 4 . À cette relative naïveté s'ajoute en 6.3 une certaine trivialité sur les analogies entre lire et écrire, l'un étant le miroir inverse de l'autre: la reprise de réflexion de la pragmatique et de la linguistique textuelle ne fait pas avancer la description du processus de traduction. Tout au long de 6.2 et 6.3 , on a semblet-il confondu à dessein «text processing» et «translating»: en faire, en conclusion (6.4), deux «special cases of human information processing» relève assez de l'artifice, d'autant plus que le chapitre 7 sur information, connaissances conceptuelles et encyclopédiques, types de mémoire, etc. est complétement détaché de la traduction. Si les chapitres antérieurs étaient linguistiques, celui-ci s'oriente vers la psycholinguistique (notions de prototype, de classe, de schéma, de script, de catégorisation, de stockage et d'extraction des connaissances, etc.): les liens avec le chapitre 6 ne sont pas explicites. De nouveau, l'affirmation (p. 263) que le modèle de traitement humain de l'information a «d'importantes implications pour la compréhension des procédures d'interrogation à l'œuvre dans la traduction» reste à démontrer! Trop «d'évidences» et de «il est clair» (pp. 237, 238, 243,249 , etc.) viennent entacher l'exposé, déjà marqué par des assertions catégoriques (exemple pp. 22-23) sur ce qu'il faut attendre des théories.

Définir le traducteur comme sujet où convergent divers types de connaissances et de compétences, notamment discursives et stratégiques (pp. 40-42), référer au contexte, à la situation d'énonciation (pp. 110-114), aux variables sociolinguistiques (pp. 184-195)... c'est se rappeler que la traduction est communication. Mais quelle est la pertinence de ce rappel si on n'en tire aucune conséquence pour théoriser? Hatim et Mason n'ont pas séparé aussi rudement intentionalité, type de texte, intertextualité, coopération, registres... Leur orientation plutôt sociolinguistique a tempéré leur ardeur sémiotique et textuelle.

Avec Bell, la traduction est perçue comme opération (psycho)linguistique et non comme acte interlinguistique et interculturel, le traducteur est caricaturé comme systèmeexpert (pp. 39-40), non comme médiateur. Ces choix et ces préférences permettent de croire que la signification des textes peut être capturée selon divers schémas et règles et transférée sans problèmes d'équivalence (pp. 6-9) ni d'effet sur les récepteurs potentiels. Un modèle du processus de traduction peut-il faire l'impasse sur les enjeux de la décision de traduire, sur l'inscription sociale de la traduction, sur les contraintes de travail du traducteur, lecteur autant que producteur de textes?

Quelle place faut-il, en dernier ressort, accorder au livre dont on vient de rendre compte? L'appareil descriptif de la théorie systémique du langage (Halliday), les concepts de De Beaugrande et de Dressler, nombre de notions de pragmatique... ne sont pas mis au service d'un portrait exclusif de la traduction. Les parties II et III ne recoupent pas systématiquement les exigences d'une théorie (posées pp. 26-28). A-t-on alors un manuel de linguistique pour traducteurs (les interprètes professionnels sont complètement exclus de l'ouvrage) et non un essai sur une «approche intégrée, interdisciplinaire, pluriméthodologique.... (p. 28)?

Après lecture, on peut avoir conscience des difficultés d'une intégration éventuelle - surtout lorsqu'il n'y a pas reconnaissance d'une relative autonomie de l'une des deux disciplines (traduction) et que l'autre est donnée comme cadre assimilateur, sinon annexionniste (linguistique appliquée) qui embrasserait la lexicologie, la pathologie du langage, l'aménagement linguistique... et la communication interlinguistique (p. 28). En tout cas, en dépit du sous-titre, théorie et pratique ne se sont guère rapprochées, au bout de ces 270 pages. L'ouvrage ne prend pas place dans les controverses actuelles sur le développement 
d'une théorie de la traduction: sa bibliographie (pp. 275-285) reflète d'ailleurs ses préférences plus linguistiques que traductologiques.

En 1965, Catford voyait la traduction à partir des catégories d'une théorie générale des langues et faisait d'elle une branche de la linguistique comparée. En 1991, Bell considère la traduction à partir des catégories d'une orientation plutôt pragmatique et psychologique de la linguistique pour tenter d'en faire une branche de la linguistique cognitive. Dans les deux cas, la ruse a consisté à esquiver l'originalité, les spécificités de la traduction, à n'en pas interroger le statut possible dans l'ensemble des sciences et des pratiques de la communication.

YVES GAMBIER Université de Turku, Turku, Finlande 